\title{
Spin state and exchange in the quasi-one-dimensional antiferromagnet $\mathrm{KFeS}_{2}$
}

\author{
SATISH KUMAR TIWARY and SUKUMARAN VASUDEVAN \\ Department of Inorganic and Physical Chemistry, Indian Institute of Science, Bangalore \\ 560012 , India
}

MS received 10 January 1997; revised 24 March 1997

\begin{abstract}
We report the optical spectra and single crystal magnetic susceptibility of the one-dimensional antiferromagnet $\mathrm{KFeS}_{2}$. Measurements have been carried out to ascertain the spin state of $\mathrm{Fe}^{3+}$ and the nature of the magnetic interactions in this compound. The optical spectra and magnetic susceptibility could be consistently interpreted using a $S=\frac{1}{2}$ spin ground state for the $\mathrm{Fe}^{3+}$ ion. The features in the optical spectra have been assigned to transitions within the $d$-electron manifold of the $\mathrm{Fe}^{3+}$ ion, and analysed in the strong field limit of the ligand field theory. The high temperature isotropic magnetic susceptibility is typical of a low-dimensional system and exhibits a broad maximum at $\sim 565 \mathrm{~K}$. The susceptibility shows a well defined transition to a three dimensionally ordered antiferromagnetic state at $T_{N}=250 \mathrm{~K}$. The intra and interchain exchange constants, $J$ and $J^{\prime}$, have been evaluated from the experimental susceptibilities using the relationship between these quantities, and $\chi_{\max }, T_{\max }$, and $T_{N}$ for a spin $\frac{1}{2}$ one-dimensional chain. The values are $J=-440.71 \mathrm{~K}$, and $J^{\prime}=53.94 \mathrm{~K}$. Using these values of $J$ and $J^{\prime}$, the susceptibility of a spin $\frac{1}{2}$ Heisenberg chain was calculated. A non-interacting spin wave model was used below $T_{\mathrm{N}}$. The susceptibility in the paramagnetic region was calculated from the theoretical curves for an infinite $S=\frac{1}{2}$ chain. The calculated susceptibility compares well with the experimental data of $\mathrm{KFeS}_{2}$. Further support for a one-dimensional spin $\frac{1}{2}$ model comes from the fact that the calculated perpendicular susceptibility at $0 \mathrm{~K}\left(2.75 \times 10^{-4} \mathrm{emu} / \mathrm{mol}\right)$ evaluated considering the zero point reduction in magnetization from spin wave theory is close to the projected value $\left(2.7 \times 10^{-4} \mathrm{emu} / \mathrm{mol}\right)$ obtained from the experimental data.
\end{abstract}

Keywords. Low-dimensional materials; magnetic ordering and exchange; spin-waves.

PACS Nos $75 \cdot 10 ; 75 \cdot 30 ; 78 \cdot 40$

\section{Introduction}

$\mathrm{KFeS}_{2}$, a linear chain insulating antiferromagnet and one of the earliest reported ternary alkali metal thioferrates [1] has been widely studied both as a model onedimensional magnetic system and as a spin analogue of the iron-sulfur ferrodoxin proteins. These proteins and $\mathrm{KFeS}_{2}$ share a common structural motif - chains of edge sharing $\left[\mathrm{FeS}_{4}\right]$ tetrahedra. $\mathrm{KFeS}_{2}$ crystallizes in $\mathrm{C} 2 / \mathrm{c}$ monoclinic space group and the chains are separated by potassium atoms (figure 1). The interchain $\mathrm{Fe}-\mathrm{Fe}$ distance $(6.6 \AA)$ is much larger than the intrachain $\mathrm{Fe}-\mathrm{Fe}$ distance $(2 \cdot 7 \AA)$ and so, magnetically, the compound has a pronounced one-dimensional character [2].

Extensive ${ }^{57} \mathrm{Fe}$ Mössbauer [3-10], neutron diffraction [11-13], magnetic susceptibility $[2,12-15]$ and optical spectroscopic measurements [16-18] on $\mathrm{KFeS}_{2}$ have been 


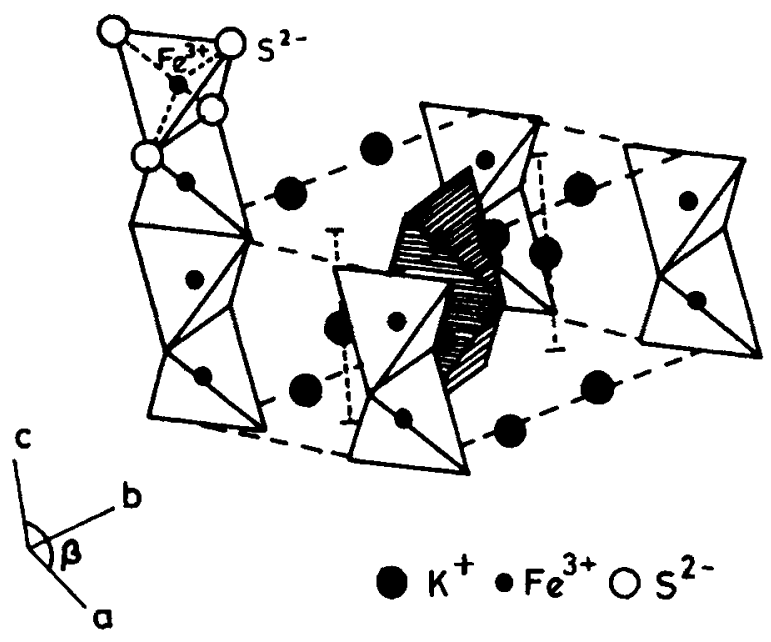

Figure 1. C 2/c crystallographic structure of $\mathrm{KFeS}_{2}$. The lattice parameters are $(a=7 \cdot 082, b=11 \cdot 329, c=5 \cdot 403, \beta=113 \cdot 2)$.

reported. The results and their interpretation, however, differ greatly from author to author. Neutron diffraction studies have shown that below $T_{N}=250 \mathrm{~K}$, the $\mathrm{Fe}^{3+}$ spins are antiferromagnetically coupled along the chain and form ferromagnetic sheets in the $a b$ plane. Powder magnetic susceptibility studies, however, with the exception of one [14], have failed to reveal any anomaly at $T=250 \mathrm{~K}$. One study reported $T_{N}=12.5 \mathrm{~K}$ [15]. The reports on the powder magnetic susceptibility by different investigators are conflicting, that it is temperature-independent $[2,13]$ or that it increases with decrease in temperature below $250 \mathrm{~K}[12,15]$ in contrast to the behaviour expected of an antiferromagnet. There are similar discrepancies in the reported values of the effective magnetic moment, $\mu_{\mathrm{eff}}$, which ranges from $0.8 \mu_{\mathrm{B}}$ [14] to $4.6 \mu_{\mathrm{B}}$ [3]. A recent single crystal magnetic susceptibility report [19], however, showed a clear antiferromagnetic transition at $250 \mathrm{~K}$, and a value of the magnetic moment expected for an $S=1 / 2$ ground state for the $\mathrm{Fe}^{3+}$ ion.

Although the optical spectra reported by various groups are broadly similar, their interpretations have varied widely in their attempt at accounting for the magnetic moment obtained from magnetic susceptibility studies. An early report had assigned the features in the optical spectra to $d-d$ transitions of a high spin $d^{5} \mathrm{Fe}^{3+}$ ion [16]. Later the same bands were reinterpreted as ligand to metal charge transfer (LMCT) on the basis of a spin-polarized multiple scattering $\chi \alpha$ calculation on an $\mathrm{FeS}_{4}^{-}$unit [20]. A similar LMCT interpretation, but on the basis of a semi-emperical extended Huckel calculation has been recently proposed [18]. The cystal field model [17], however, supports an assignment based on $d-d$ transtions of a low-spin $d^{5}$ ion. The variable energy photo-electron spectra have been interpreted assuming a high spin $d^{5}$ ion with an inverted bonding scheme due to strong covalency [21].

In general, most authors have favoured the high spin ground state $\left(S=\frac{5}{2}\right)$ for the $\mathrm{Fe}^{3+}$ ion in $\mathrm{KFeS}_{2}$ and have invoked either covalency [15] or quantum spin reduction $[8,14]$ to account for the intermediate to low spin values as found in susceptibility 


\section{One-dimensional antiferromagnet $\mathrm{KFeS}$}

measurements. The covalency argument, however, fails to account for the low saturation value, viz. $\sim 20 \mathrm{~T}$, of the magnetic hyperfine field in the Mössbauer spectra of $\mathrm{KFeS}_{2}$. This value may be compared with $60 \mathrm{~T}$ expected of a high spin $\mathrm{Fe}^{3+}$ ion as observed, e.g., in the one-dimensional high spin $\mathrm{K}_{2} \mathrm{FeF}_{5}$ [8]. Quantum spin reduction, although expected to be enhanced in low-dimensional systems, is unlikely to account for a reduction in the room temperature magnetic moment from $\sim 5.9 \mu_{\mathrm{B}}$ to $\sim 0.8 \mu_{\mathrm{B}}$ for the nearly classical $S=\frac{5}{2}$ ion. Thus in spite of numerous studies, the true nature of the spin state and magnetic behaviour of $\mathrm{KFeS}_{2}$ still remains unclear. With this in mind, we have carried out a detailed study of the optical spectra and an analysis of the single crystal magnetic susceptibility of $\mathrm{KFeS}_{2}$.

A major source of error and the possible origin of the discrepancies in the powder susceptibility measurements reported so far is the formation of ferromagnetic oxide impurities. This has been pointed out earlier [14]. A recent report on the crystal susceptibilities of a closely related compound, $\mathrm{TIFeS}_{2}$ [22] corroborates this view. It is known that on exposure to air $\mathrm{KFeS}_{2}$ is slowly oxidized to $\mathrm{KFeO}_{2}$, a high spin $\mathrm{Fe}^{3+}$ ferromagnet $\left(T_{\mathrm{c}}=960 \mathrm{~K}\right)$ [23]. Another source of surface oxidation is in the preparation; $\mathrm{KFeS}_{2}$ is usually grown from a carbonate flux and the crystals extracted with hot water. Even trace amounts of these oxide impurities can mask the true magnetic behaviour, especially since, as found in the present work, the susceptibility of $\mathrm{KFeS}_{2}$ is extremely small $\left(1.2 \times 10^{-6} \mathrm{emu} / \mathrm{g}\right.$ at $\left.300 \mathrm{~K}\right)$. In the present investigation scrupulous care has been taken to prevent formation of oxide impurities which could lead to erroneous results.

Our study shows that the anisotropic magnetic susceptibility of $\mathrm{KFeS}_{2}$ is typical of a one-dimensional antiferromagnet with $T_{\mathrm{N}}=250 \mathrm{~K}$. Both optical and magnetic measurements could be consistently interpreted using a $S=\frac{1}{2}$ spin ground state. The intra- and inter-chain exchange values, $J$ and $J^{\prime}$, have been evaluated from the experimental susceptibilities using the relationship between the exchange constants, and $\chi_{\max }, T_{\max }$, and $T_{\mathrm{N}}$ for a one-dimensional Heisenberg antiferromagnet. Using these values of $J$ and $J^{\prime}$, the susceptibility of a spin $\frac{1}{2}$ one-dimensional antiferromagnetic chain was calculated - below $T_{\mathrm{N}}$ from a non-interacting spin wave model [24], and at higher temperatures using a parametrized form of the Bonner-Fisher susceptibility curves [25]. The calculated susceptibilities compare extremely well with the experimental data for $\mathrm{KFeS}_{2}$. Further support for a spin $\frac{1}{2}$ one dimensional model comes from the calculated susceptibility at $0 \mathrm{~K}$ from spin wave theory, which is quite close to the projected experimental value of perpendicular susceptibility at $0 \mathrm{~K}$.

\section{Experimental}

$\mathrm{KFeS}_{2}$ was prepared by heating a $4: 1$ molar mixture of $\mathrm{K}_{2} \mathrm{CO}_{3}$ and oxide-free iron powder in a stream of dry $\mathrm{H}_{2} \mathrm{~S}$ at $1000 \mathrm{~K}$ for $24 \mathrm{~h}$. The product was cooled to ambient temperature over a period of $24 \mathrm{~h}$, and then extracted with cold water. This procedure gave fine permanganate-colored, needle-shaped crystals typically $5-10 \mathrm{~mm}$ in length and $0 \cdot 5-1 \mathrm{~mm}$ width. The needle axis is the c-axis. The crystals were again heated in flowing $\mathrm{H}_{2} \mathrm{~S}$ at $773 \mathrm{~K}$ for $12 \mathrm{~h}$. Under these conditions, $\mathrm{KFeO}_{2}$ is known to convert to $\mathrm{KFeS}_{2}[14,23]$. Care was taken not to expose the crystals to air or moisture after the $\mathrm{H}_{2} \mathrm{~S}$ treatment. It was found by EDAX (Cambridge Instruments SCM S360) 
measurements that exposing the crystals to air gave rise to an oxide film as detected by the oxygen-to-sulfur ratio.

Optical absorption spectra in $200 \mathrm{~nm}$ to $1600 \mathrm{~nm}\left(50000 \mathrm{~cm}^{-1}\right.$ to $\left.6250 \mathrm{~cm}^{-1}\right)$ range was recorded on a Hitachi U3400 spectrophotometer. The samples were finely dispersed in a high molecular weight paraffin and applied onto an optically polished quartz plate. The plate was mounted inside the sample holder of a closed cycle cryostat (air products).

Magnetic susceptibility measurements were made on a Faraday magnetic balance. Temperatures in the range $45-300 \mathrm{~K}$ were obtained using a closed cycle cryostat. Measurements in the temperature range $300 \mathrm{~K}-800 \mathrm{~K}$ were performed using a high temperature furnace assembly. The susceptometer was calibrated using $\mathrm{Hg}\left[\mathrm{Co}(\mathrm{NCS})_{4}\right]$ as a standard. A large number of needle-like crystals were aligned parallel onto a thin quartz plate using silicone grease. The plate was then suspended from the balance by an arrangement similar to that reported in [26]. For the high temperature measurements, the crystals were tightly packed and sealed under vacuum in small thin walled quartz ampoules of $0.5 \mathrm{~mm}$ wall thickness, $4 \mathrm{~mm}$ diameter and $10 \mathrm{~mm}$ length. Heating rates were $1-2 \%$ min in order to ensure thermal equilibrium of the sample with the He exchange gas. Calibration with $\mathrm{HgCo}(\mathrm{NCS})_{4}$ was repeated under identical conditions. The susceptibilities reported are for two directions. In the temperature range $250 \mathrm{~K}$ to $800 \mathrm{~K}$, the applied field is parallel and perpendicular to the c-axis, which is also the needle axis of the crystal. For temperatures below $250 \mathrm{~K}$, the field is parallel and perpendicular to the magnetization axis. The magnetization axis is known from neutron diffraction studies to be at an angle of approximately $13^{\circ}$ to the chain axis [11]. The absence of ferromagnetic impurities in the samples was confirmed by measuring the field dependence of the magnetization at the lowest temperatures. The susceptibility measurements were repeated to ensure that the data was reproducible.

\section{Results}

\subsection{Optical spectra}

The optical absorption spectra of $\mathrm{KFeS}_{2}$ recorded at $15 \mathrm{~K}$ is shown in figure 2 . With the exception of the features below $400 \mathrm{~nm}\left(25000 \mathrm{~cm}^{-1}\right)$ the spectrum is essentially temperature independent although better resolved at lower temperatures. In figure 2, the $300 \mathrm{~K}$ spectrum is shown only below $350 \mathrm{~nm}$ range. It may be seen that there is a sharpening of the intense absorptions at 227,265 and $281 \mathrm{~nm}(44055,37455$ and $\left.35585 \mathrm{~cm}^{-1}\right)$. The peaks at $815 \mathrm{~nm}\left(12270 \mathrm{~cm}^{-1}\right), 770 \mathrm{~nm}\left(12990 \mathrm{~cm}^{-1}\right)$ and $730 \mathrm{~nm}$ $\left(13700 \mathrm{~cm}^{-1}\right)$ are much better resolved in the low temperature spectra. Optical spectra of $\mathrm{KFeS}_{2}$ in the $900-200 \mathrm{~nm}$ range has been previously reported. The present spectra are, however, of much better quality, and cover a wider spectral range. The positions of the various optical features are given in table 1.

\subsection{Magnetic susceptibility}

The temperature variation of the magnetic susceptibility of $\mathrm{KFeS}_{2}$ crystals is shown in figure 3. The diamagnetic contributions have been subtracted from the data. The van Vleck paramagnetic contribution was evaluated from the EPR $g$ value of 2.025 [27] 


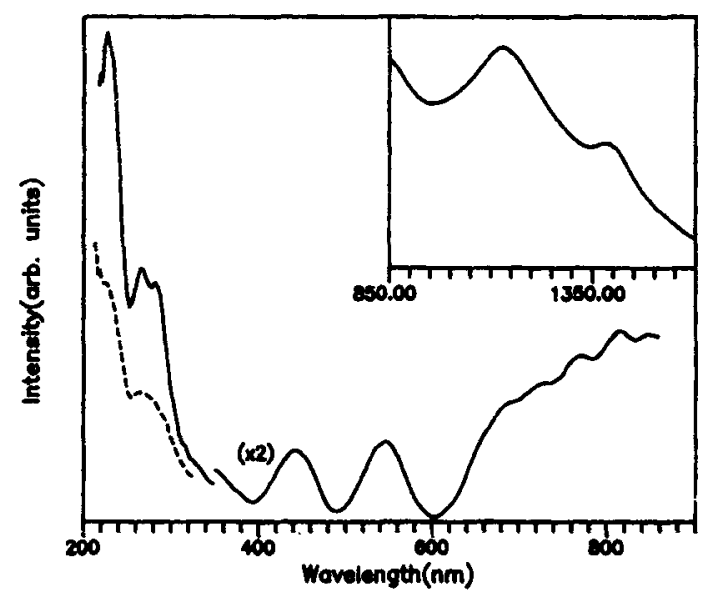

Figure 2. The optical absorption spectra of $\mathrm{KFeS}_{2}$ at $15 \mathrm{~K}$. The inset shows the spectra in the near infrared region $(850-1600 \mathrm{~nm})$. The dashed line is the spectra at $300 \mathrm{~K}$.

Table 1. Calculated and observed energies for the optical transitions in $\mathrm{KFeS}_{2}$ and their assignments.

\begin{tabular}{|c|c|c|}
\hline \multicolumn{2}{|c|}{ Energy $\left(\mathrm{cm}^{-1}\right)$} & \multirow[b]{2}{*}{ Assignment } \\
\hline Experimental & Calculated & \\
\hline 7150 & 7535 & ${ }^{2} T_{2} \rightarrow{ }^{4} T_{2}$ \\
\hline 8770 & 8780 & $\rightarrow{ }^{6} A_{1}$ \\
\hline 11765 & 11750 & $\rightarrow{ }^{4} T_{1}$ \\
\hline 12270 & 12485 & $\rightarrow{ }^{2} A_{2}$ \\
\hline 12990 & 13004 & $\rightarrow{ }^{2} T_{1}$ \\
\hline 13700 & 13341 & $\rightarrow{ }^{2} T_{2}$ \\
\hline \multicolumn{3}{|l|}{14495} \\
\hline \multirow[t]{3}{*}{18180} & 17685 & $\rightarrow{ }^{2} E$ \\
\hline & 20285 & $\rightarrow{ }^{2} T_{1}$ \\
\hline & 20818 & $\rightarrow{ }^{2} T_{2}$ \\
\hline 22470 & 22885 & $\rightarrow{ }^{2} A_{1}$ \\
\hline 35587 & & Charge transfer \\
\hline 37735 & & Charge transfer \\
\hline 44053 & & Charge transfer \\
\hline
\end{tabular}

and the energies of the optical transitions; its value is $9 \times 10^{-7} \mathrm{emu} / \mathrm{mol}$. The susceptibility of $\mathrm{KFeS}_{2}$ is typical of a low-dimensional antiferromagnet undergoing a threedimensional transition. The Neel temperature is $250 \mathrm{~K}$. In figure 3, the data above $250 \mathrm{~K}$ is the susceptibility parallel and perpendicular to the c-axis and is isotropic within the experimental limits. The high temperature isotropic susceptibility shows a broad maximum at $565 \mathrm{~K}\left(T_{\max }=565 \mathrm{~K}, \chi_{\max }=2.7 \times 10^{-4} \mathrm{emu} / \mathrm{mol}\right)$, and even at the highest temperature, does not show Curie-Weiss behaviour. The low temperature $(T<250 \mathrm{~K})$ behaviour is typical of antiferromagnetic ordering where the susceptibility 


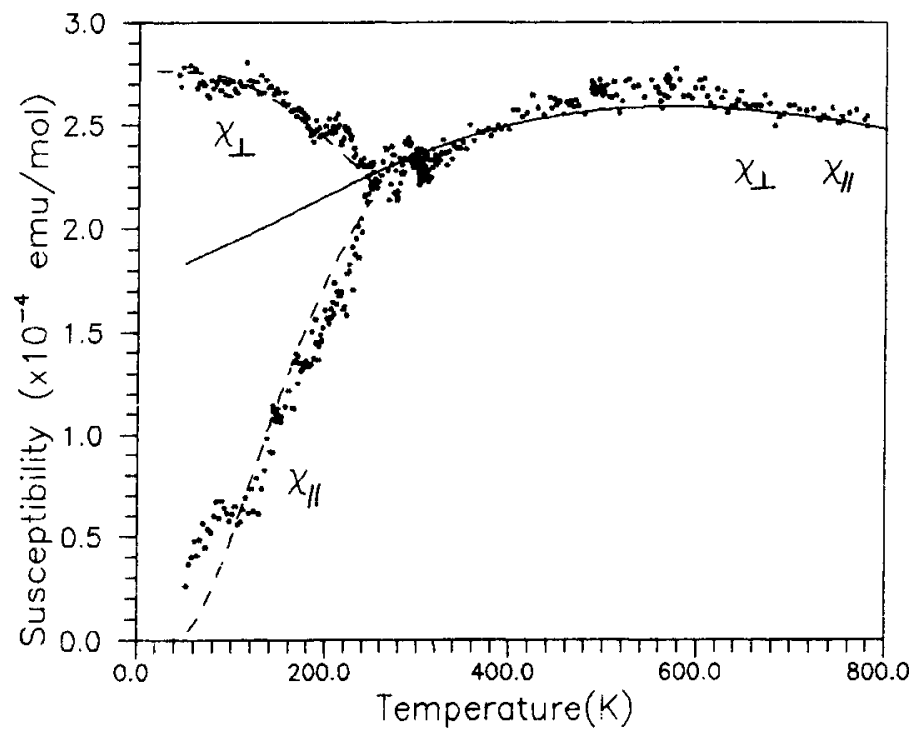

Figure 3. The experimental magnetic susceptibility of $\mathrm{KFeS}_{2}$. Above $250 \mathrm{~K}$, the external field is applied parallel and perpendicular to the c-axis. Below $250 \mathrm{~K}$, the field is parallel and perpendicular to the magnetization axis. The solid line is the calculated susceptibility using eq. (5). The dashed lines are the susceptibility calculated in the spin wave aproximation - the parallel susceptibility using eq. (9) and the perpendicular susceptibility using eq. (13). The calculated susceptibilities over the whole temperature range are for $J=-440.71 \mathrm{~K}, J^{\prime}=53.94 \mathrm{~K}, g=2.025$, and $S=\frac{1}{2}$.

parallel to the magnetization axis shows a sharp decrease below $T_{\mathrm{N}}$ whereas the perpendicular susceptibility shows only a weak temperature dependence. The extrapolated value of the perpendicular susceptibility $\left(\chi_{\perp}\right)$ of $\mathrm{KFeS}_{2}$ at $0 \mathrm{~K}$ is $2.7 \times 10^{-4} \mathrm{emu} / \mathrm{mol}$. The present single crystal susceptibility value of $2.3 \times 10^{-4} \mathrm{emu} / \mathrm{mol}$ at $300 \mathrm{~K}$ is much lower than the previously reported powder susceptibility values, with the exception of ref. [14], confirming substantial impurity contribution in their powder susceptibility data.

\section{Discussion}

\subsection{Optical spectra}

The optical spectra in the $400-1500 \mathrm{~nm}\left(25000-6667 \mathrm{~cm}^{-1}\right)$ region have been interpreted in terms of transitions within the $d$-electron manifold of a low spin $\mathrm{Fe}^{3+}$ ion in a tetrahedral crystal field. The absence of temperature shifts constitutes strong evidence for these features being internal crystal field transitions. The temperature dependent features in the ultraviolet part of the spectrum probably have their origin in charge transfer. An a priori justification for assuming a low-spin ground state comes from the low value of the magnetic susceptibility (figure 3 ). The $d-d$ transitions were analysed in the strong field limit of crystal field theory [28]. In this limit, the crystal field is 


\section{One-dimensional antiferromagnet $\mathrm{KFeS}_{2}$}

considered to be much stronger than the electronic repulsion, which is treated as a perturbation. The small tetragonal distortion of the $\mathrm{FeS}_{4}$ tetrahedra has been neglected, due to the spin-orbit coupling, since the value of $g$ obtained from EPR studies is 2.025 [27]. The energies were obtained by diagonalization of the appropriate strong field matrices after allowing for full interconfigurational mixing [28]. The energies of the metal ion states are a function of three variables $-D q$, the crystal field splitting energy, and $B$ and $C$, the Raccah inter-electron repulsion parameters. $B$ and $C$ are related to the Slater-Condon parameters $F_{2}$ and $F_{4}$ by the linear relation [28], $C=5 F_{4}$ and $B=F_{2}-5 F_{4}$. The best fit between the experimental and calculated values were obtained for $D q=1410 \mathrm{~cm}^{-1}, B=520 \mathrm{~cm}^{-1}$ and $C=1285 \mathrm{~cm}^{-1}$. The experimental and calculated values alongwith their assignments are given in table 1 . Attempts to fit the spectra using the high spin ${ }^{6} A_{1}$ as the ground state were unsuccessful for reasonable values of the crystal field parameters.

The features in the near infrared region at $7150 \mathrm{~cm}^{-1}$ and $8770 \mathrm{~cm}^{-1}$ have been assigned to the spin forbidden ${ }^{2} T_{2} \rightarrow{ }^{4} T_{1}$ and ${ }^{2} T_{2} \rightarrow{ }^{6} A_{1}$ transitions. A possible reason for the intensity of these spin forbidden bands is that exchange interactions can relax the spin selection rules without energetically perturbing the states involved. A similar explanation had been proposed for the anomalously high intensities of the spin forbidden bands in the optical spectra of one-dimensional compounds $\mathrm{RbFeX}$ $(X=\mathrm{Cl}$ or $\mathrm{Br})$ [29]. It was found that in these compounds the intensity of such exchange allowed bands does not show any temperature dependence. Nor is the intensity enhancement related to the three-dimensional ordering temperature. A similar behaviour is observed in the case of $\mathrm{KFeS}_{2}$.

\subsection{Magnetism}

4.2.1 Exchange interactions: The magnetic susceptibility data of $\mathrm{KFeS}_{2}$ is typical of a low-dimensional system. The broad maximum at $565 \mathrm{~K}$ and the non-Curie-Weiss behaviour are due to the short range spin correlations which manifest at temperatures much above $T_{\mathrm{N}}$ in low-dimensional systems. The $T_{\mathrm{N}}$ is, of course, subject to the interchain interactions which lead to three-dimensional ordering below $250 \mathrm{~K}$. The low value of magnetic susceptibility and its isotropic behaviour above $T_{\mathrm{N}}$ suggest that $\mathrm{KFeS}_{2}$ may be modelled as a spin- $\frac{1}{2}$ one-dimensional Heisenberg chain. Further support comes from the experimental value of the quantity $\chi_{\max } T_{\max } / g^{2}$ which is a handy criterion for determining the spin state appropriate for the one dimensional chain compound under investigation. For a one-dimensional Heisenberg chain the values of this quantity for various spin states are 0.0353 for $S=1 / 2 ; 0.089$ for $S=1 ; 0 \cdot 16$ for $S=3 / 2 ; 0.25$ for $S=2$; and 0.38 for $S=5 / 2$ [30]. From the experimentally measured susceptibility of $\mathrm{KFeS}_{2}$ (figure 3 ) we have $T_{\max }=565 \mathrm{~K}$ and $\chi_{\max }=2.7 \times 10^{-4} \mathrm{emu} / \mathrm{mol}$. Using the EPR determined value of $g=2.025$ [27] one obtains

$$
\frac{\chi_{\max } T_{\max }}{g^{2}}=0.0375
$$

which is closest to the value for $S=1 / 2$ chain.

The moment values reported from the neutron scattering studies are, however, much higher $(\mu=2 \cdot 4$ [11], $2 \cdot 3$ [12], and 1.9 [13]). It is, however, difficult to reconcile the 
present magnetic studies with higher spin states for $\mathrm{Fe}^{3+}$ since the experimental value of $\chi_{\max } T_{\max } / g^{2}$ clearly indicates a $S=1 / 2$ state. Accordingly, we have evaluated the exchange constants: $J$, the intra-chain nearest-neighbour exchange, and $J^{\prime}$, the interchain exchange using the properties of spin $\frac{1}{2}$ AFM Heisenberg chains.

According to Bonner and Fisher $[30,31]$, for a spin $\frac{1}{2}$ chain of infinite length,

$$
\frac{k_{\mathrm{B}} T_{\max }}{|J|}=1 \cdot 282
$$

and

$$
\frac{|J| \chi_{\max }}{N g^{2} \mu_{\mathrm{B}}^{2}}=0.07346
$$

Substituting the experimental value of $T_{\max }, 565 \mathrm{~K}$, in eq. (1), we arrive at a $|J|$ value of $440.71 \mathrm{~K}$. Putting this value of $|J|$ in eq. (2) and using the EPR-determined value of $g=2.025$ [27], we get a value of $\chi_{\max }=2.5 \times 10^{-4} \mathrm{emu} / \mathrm{mol}$. This value is a little less than the experimental value of $2.7 \times 10^{-4} \mathrm{emu} / \mathrm{mol}$.

This discrepancy in the calculated and experimental values is probably due to the interchain coupling, which is known to be ferromagnetic. The interchain exchange, $J^{\prime}$, was calculated from $T_{\mathrm{N}}$ using a relationship originally due to Oguchi, who had calculated the ratio of the exchange parameters, $J / J^{\prime}$, from $T_{\mathrm{N}}$ by a Green's function technique [32]

$$
\frac{k_{\mathrm{B}} T_{\mathrm{N}}}{|J|}=\frac{4 S(S+1)}{3 I_{\left(J / J^{\prime}\right)}} \text {. }
$$

The integral $I_{J / J}$, has been numerically evaluated. Substituting the values of $T_{\mathbf{N}},|J|$, and $S$ in eq. (3), the value of the integral $I_{\left(J / J^{\prime}\right)}$ is $1 \cdot 8$, and $\left|J^{\prime}\right|=53.94 \mathrm{~K}$.

In order to evaluate how good a spin- $\frac{1}{2}$ one-dimensional antiferromagnetic system is in modelling the magnetic properties of $\mathrm{KFeS}_{2}$, we have calculated the susceptibility of such a model chain for $|J|=440.71 \mathrm{~K}, J^{\prime}=53.94 \mathrm{~K}, g=2.025$ and $S=\frac{1}{2}$ and compared it with the experimental magnetic susceptibility of $\mathrm{KFeS}_{2}$, both above and below $T_{\mathrm{N}}$.

4.2.2 Paramagnetic susceptibility: The high temperature paramagnetic susceptibility was calculated from the susceptibility curves of Bonner and Fisher [31] using a parametrization due to Torrence et al [25]

$$
\begin{aligned}
\chi_{\text {chain }} & =\frac{1.4892}{|J|} \cdot\left[0.05031+0.013014 x+0.046846 x^{2}-0.062319 x^{3}\right. \\
& \left.+0.030834 x^{4}-0.0076763 x^{5}+0.00096332 x^{6}-0.000048529 x^{7}\right]
\end{aligned}
$$

where $x=T /|J|$.

Interchain interactions were treated in the mean field approximation [33]

$$
\chi=\frac{\chi_{\text {chain }}}{\left[1-\left(2 z j^{\prime} / N g^{2} \mu_{\mathrm{B}}^{2}\right) \chi_{\text {chain }}\right]} .
$$

Equation (5) was evaluated at various temperatures for $|J|=440 \cdot 71 \mathrm{~K}$, $z j^{\prime}=J^{\prime}=53.94 \mathrm{~K}$, and $g=2.025$. The calculated values are shown as the solid curve in figure 3. It may be seen that the agreement between the calculated and experimental values in the paramagnetic region, $300 \mathrm{~K}$ to $800 \mathrm{~K}$, is extremely good. 


\section{One-dimensional antiferromagnet $\mathrm{KFeS}_{2}$}

4.2.3 Low temperature susceptibility: The susceptibility below $T_{\mathrm{N}}$ was calculated using a simple one-dimensional non-interacting spin wave model [24]. It might at first seem surprising that such a model would be appropriate for the low-temperature threedimensional AFM state of $\mathrm{KFeS}_{2}$. The justification comes from neutron scattering results [34] which showed that magnon dispersion along the $a^{*}$ and $b^{*}$ axes was very much weaker than that along the chain direction.

The effective Hamiltonian for such a system is given by

$$
\mathscr{H}=\sum_{i, j} J_{i, j} S_{i} S_{j}+\mathscr{D} \sum_{i} S_{i}^{2}-g \mu_{\mathrm{B}} H_{0} \sum_{i} S_{i}
$$

The interchain interactions have been included as an anisotropy term, $\mathscr{D}$,

$$
\mathscr{D}=J^{\prime}=g \mu_{\mathrm{B}} H_{\mathrm{A}},
$$

where $H_{\mathrm{A}}$, is the anisotropic field felt by each ion. The magnetization of the antiferromagnetically ordered state was modelled using a two sublattice model. Magnon energies were calculated using the Holstein-Primakoff approximation and considering only nearest neighbour interactions along the chain axis [24]. The effective Hamiltonian (eq. (6)) thus gives rise to the following grand partition function

$$
\begin{aligned}
\Xi= & \exp \left(-E_{0} / k_{\mathrm{B}} T\right) \exp \left(\sum_{k}\left[\beta_{k}-A k_{\mathrm{B}} T\right]\right) \\
& \times \prod_{k}\left[1-\exp \left(-\beta_{k}+\beta_{\mathrm{H}}\right)\right]\left[1-\exp \left(-\beta_{k}-\beta_{\mathrm{H}}\right)\right],
\end{aligned}
$$

where $k$ is restricted to a one-dimensional Brillouin zone since magnon dispersion in the other directions is weak [34]. In the above equation

$$
E_{0}=-2 N_{0}(\mathscr{D}+J),
$$

where $N_{0}$ is the number of magnetic atoms in each sublattice,

$$
\begin{aligned}
\beta_{k} & =\left(A^{2}-\left|B_{k}\right|^{2}\right)^{1 / 2} / k_{\mathrm{B}} T, \\
\beta_{\mathrm{H}} & =g \mu_{\mathrm{B}} H_{0} f / k_{\mathrm{B}} T, \\
A & =\mathscr{D}+2|J|, \\
B_{k} & =2|J| \cos (\mathbf{k} . \mathrm{a}),
\end{aligned}
$$

where $\mathrm{a}$ is the interatomic distance between the $\mathrm{Fe}^{3+}$ ions in the chain, and

$$
f=\left(1-\chi_{\|} / 2 \chi_{1}\right)
$$

is a correction factor coming from higher order effects [24].

4.2.4 Parallel susceptibility: The parallel susceptibility is given by

$$
\chi_{\|}=N k_{\mathrm{B}} T \cdot \frac{\mathrm{d}^{2} \ln \Xi}{\mathrm{d} H_{0}^{2}}
$$


which gives, in the present case,

$$
\chi_{\|}=\frac{N\left(g \mu_{\mathrm{B}}\right)^{2}}{k_{\mathrm{B}} T} \sum_{-\pi / a}^{\pi / a} \frac{\exp \left(\beta_{k}\right)}{\left[1-\exp \left(\beta_{k}\right)\right]^{2}} .
$$

Equation (9) was evaluated at various temperatures for $|J|=440.71 \mathrm{~K}$ and $\mathscr{D}=53.94 \mathrm{~K}$. The correction factor was calculated from the experimental value of $\chi_{\|}$and the extrapolated value of $\chi_{\perp}$ at $0 \mathrm{~K}$. The calculated susceptibility is shown as the dashed curve in figure 3. It may be seen that the agreement with the experimental susceptibility of $\mathrm{KFeS}_{2}$ is reasonably good between $T_{\mathrm{N}}$ and $\approx 100 \mathrm{~K}$. At lower temperatures, the agreement is slightly poorer.

4.2.5 Perpendicular susceptibility: In spin wave theory, the perpendicular susceptibility is derived [24] as

$$
\chi_{\perp}=\frac{\chi_{\perp}^{0}}{(1+\alpha / 2)}\left[1-\frac{\Delta M_{s p}}{M_{s 0}}-\frac{\Delta M_{s T}}{M_{s 0}}\right],
$$

where $\chi_{\perp}^{0}$ is the perpendicular susceptibility as given by the mean field approximation; $\alpha$, the anisotropy factor, is $g \mu_{\mathrm{B}} H_{\mathrm{A}} / 2 S z|J|$, which for the present one-dimensional system is $\mathscr{D} / 2|J|=0.0612 . M_{s 0}$ is the fully aligned sublattice magnetization; $\Delta M_{s \beta}$ is the zero point deviation from the completely aligned state and is independent of temperature, and $\Delta M_{s T}$ is the deviation at finite temperatures.

At absolute zero,

$$
\chi_{\perp}(0)=\frac{\chi_{\perp}^{0}}{(1+\alpha / 2)} \cdot\left[1-\frac{\Delta M_{s \beta}}{M_{s 0}}\right] .
$$

$\chi_{\perp}^{0}$, the mean field perpendicular susceptibility is, for $J=440.71 \mathrm{~K}$ and $J^{\prime}=53.94 \mathrm{~K}$, $4 \cdot 11 \times 10^{-4} \mathrm{emu} / \mathrm{mol}$.

According to the spin wave theory, the zero point deviation in magnetization, $\Delta M_{s \beta} / M_{s 0}$, for a spin $\frac{1}{2}$ linear Heisenberg system is

$$
\frac{\Delta M_{s \beta}}{M_{s 0}}=-\frac{1}{2 S}\left(1+\frac{\ln 2 \alpha}{\pi}\right)
$$

which, for $\alpha=0.061$ and $S=\frac{1}{2}$ is 0.3304 . Substituting the values of $\chi_{\perp}^{0}, \alpha$, and $\Delta M_{s \beta} / M_{s 0}$ in eq. (11) we get $\chi_{\perp}(0)=2.75 \times 10^{-4} \mathrm{emu} / \mathrm{mol}$. This value may be compared with the extrapolated experimental value of $2.7 \times 10^{-4} \mathrm{emu} / \mathrm{mol}$.

The temperature dependence of the perpendicular susceptibility in the spin wave approximation is given by the relation

$$
\chi_{\perp}(T)=\chi_{\perp}(0)-\frac{\chi_{\perp}^{0}}{(1+\alpha / 2)}\left[\frac{\Delta M_{s T}}{M_{s 0}}\right] .
$$

For small value of the applied field, the $H_{0}$ term may be dropped and $\Delta M_{s T} / M_{\text {so }}$ approximated as

$$
\frac{\Delta M_{s T}}{M_{s 0}}=\frac{A \mathbf{a}}{\pi k_{\mathbf{B}} T} \int\left[\beta_{k}\left(\exp \left(\beta_{k}-1\right)\right)\right]^{-1} \mathrm{~d} k .
$$


Equation (14) was evaluated for $J=-440.71 \mathrm{~K}$ and $\mathscr{D}=53.94 \mathrm{~K}$, and the perpendicular susceptibility calculated from eq. (13). The calculated susceptibilities are shown as dashed curves in figure 3. It may be seen that as in the case of parallel susceptibility, the calculated and experimental values compare reasonably well.

\section{Concluding remarks}

The present optical spectroscopy and single crystal magnetic susceptibility measurements on the linear chain antiferromagnet $\mathrm{KFeS}_{2}$ confirm an $S=\frac{1}{2}\left({ }^{2} T_{2}\right)$ ground state for the $\mathrm{Fe}^{3+}$ ion and that the magnetic interactions are essentially one-dimensional in nature. Three-dimensional antiferromagnetic ordering sets in at $T_{\mathrm{N}}=250 \mathrm{~K}$. The features in the optical spectra have been assigned to $d-d$ transitions within the $3 d$ electron manifold of the $\mathrm{Fe}^{3+}$ ions, and there is reasonable agreement with the transition energies as calculated from the strong field limit of the ligand field theory for the crystal field parameter $D q=1410 \mathrm{~cm}^{-1}$, and the Raccah interelectronic repulsion parameters $B=520 \mathrm{~cm}^{-1}$ and $C=1285 \mathrm{~cm}^{-1}$. The magnetic susceptibility data of $\mathrm{KFeS}_{2}$ is typical of a low-dimensional system. The susceptibility shows a broad maximum at $565 \mathrm{~K}$ and non-Curie-Weiss behaviour even at very high temperatures. There is a well-defined transition at $250 \mathrm{~K}$, below which the susceptibility is typical of a three-dimensional antiferromagnet. We believe that the present results - the first report of the crystal susceptibility of $\mathrm{KFeS}_{2}$-are superior to most of the previous powder results even in the high temperature isotropic region since extreme care has been taken to eliminate ferromagnetic oxide impurities which appear to have plagued previous results $[2,12,13]$.

The intra- and inter-chain exchange constants, $J$ and $J^{\prime}$ were evaluated from the relationship between these quantities and $\chi_{\max }, T_{\max }$ and $T_{\mathrm{N}}$ for a spin- $\frac{1}{2}$ one-dimensional Heisenberg system. The values of $J$ and $J^{\prime}$ are $-440.71 \mathrm{~K}$ and $53.94 \mathrm{~K}$. Using these values the susceptibility of a spin- $\frac{1}{2}$ Heisenberg chain was calculated at low temperature using a non-interacting spin wave model, and at high temperatures using a parametrized form of the Bonner-Fisher curves. The calculated and experimental susceptibilities compare extremely well. Further support for a $S=\frac{1}{2}$ model comes from the fact that the calculated perpendicular susceptibility at $0 \mathrm{~K}\left(2.75 \times 10^{-4} \mathrm{emu} / \mathrm{mol}\right)$ evaluated considering the zero point reduction from the spin wave theory is extremely close to the projected value obtained from the experimental data $\left(2.7 \times 10^{-4} \mathrm{emu} / \mathrm{mol}\right)$. The low value of susceptibility in $\mathrm{KFeS}_{2}$ is adequately explained by quantum spin reduction for an $S=\frac{1}{2}$ chain and there is no need to invoke covalency as has been done recently for $\mathrm{TlFeS}_{2}[22]$.

The ratio $J^{\prime} / 2 J=0.0612$ is rather high for a one-dimensional system. This value, however, is dictated by the fact that the experimental values, $T_{\max }$ and $T_{\mathrm{N}}$, are themselves high. The low temperature susceptibility could, however, still be modelled by a $S=\frac{1}{2}$ one-dimensional antiferromagnetic chain since magnon dispersion in non-chain directions is comparatively weak. Interchain interactions may hence be adequately represented by an anisotropy field, and the one-dimensional spin wave model retained.

\section{Acknowledgement}

One of the authors (SKT) thanks CSIR, New Delhi, India for a research fellowship. 


\section{References}

[1] W Bronger, Angew. Chem. Int. Ed. 20, 52 (1981)

[2] W Bronger, Z. Anorg. Allg. Chem. 359, 225 (1968)

[3] D Raj and S P Puri J. Chem. Phys. 50, 3184 (1969)

[4] C A Taft, D Raj and J Danon, J. Phys. 35, C6-241 (1974)

[5] C A Taft, J. Phys. 38, 1161 (1977)

[6] R B Scorzelli, C A Taft, J Danon and V K Garg, J. Phys. C11, 1397 (1978)

[7] T P Arsenio, P H Domigues, N C Furtado and C A Taft, Solid State Commun. 38, 205(1981)

[8] D M Cooper, D P E Dickson, P H Domingues, C P Gupta, C E Johnson, M F Thomas, C A Taft and P J Walker, J. Magn. Magn. Mater. 36, 171 (1983)

[9] J Zink and K Nagorny, J. Phys. Chem. Solids 491429 (1988)

[10] L Cianchi, F Del Giallo and F Pieralli, J. Phys. C21, 2931 (1988)

[11] M Nishi and Y Ito, Solid State Commun. 30, 571 (1979)

[12] Z Tomkowicz, A Szytula and H Bak-Ptasiewicz, Phys. Status Solidi 57, K25 (1980)

[13] W Bronger, A Kyas and P Muller, J. Solid State Chem. 70, 262 (1987)

[14] D C Johnston, S C Mraw and A J Jacobson, Solid State Commun. 44, 255 (1982)

[15] A Mauger, M Escorne, C A Taft, N C Fưrtado, Z P Arguello and T P Arsenio, Phys. Rev. B30, 5300 (1984)

[16] C A Taft and M A de Paoli, Chem. Phys. Lett. 68, 94 (1979)

[17] H H Schmidtke, R Packrof,, W Bronger and P Muller, Chem. Phys. Lett. 150, 129 (1988)

[18] R Packroff and H H Schmidtke, Inorg. Chem. 32, 654 (1993)

[19] S K Tiwary and S Vasudevan, Solid State Commun. 101, 449 (1997)

[20] C A Taft and M Braga, Phys. Rev. B21, 5802 (1980)

[21] K D Butcher, G S Matthew and E I Solomon, Inorg. Chem. 29, 2067 (1990)

[22] D Welz and M Nishi, Phys. Rev. B45, 9806 (1992)

[23] A J Jacobson, M S Whittingham and S M Rich, J. Electrochem. Soc. 126, 887 (1979)

[24] F Keffer, in Handbuch der Physik edited by S Flügge (Springer-Verlag, Berlin, 1966) vol. XVIII/2, pp. 1-273, and references therein

[25] J B Torrance, Y Tomkiewicz and B D Silverman, Phys. Rev. B15, 4738 (1987)

[26] D A Cruse and M Gerloch, J. Chem. Soc. Dalton Trans. (1977) 152

[27] W V Sweeney and R E Coffman, Biochim. Biophys. Acta 286, 26 (1972)

[28] J S Griffith, Theory of transition metal ions (Cambridge University Press, Cambridge, England, 1961)

[29] C F Putnik, G M Cole Jr., B B Garrett and S L Holt, Inorg. Chem. 15, 826 (1976)

[30] L D de Jongh and A R Miedema, Adv. Phys. 23, 1 (1974)

[31] J C Bonner and M E Fisher, Phys. Rev. 135, A640 (1964)

[32] T Oguchi, Phys. Rev. 133, A1098 (1964)

T Oguchi and A Honma, J. Appl. Phys. 34, 1153 (1963)

[33] J S Smart, Effective field theories of magnetism (Saunders, Philadelphia, 1986)

[34] M Nishi, Y Ito and S Funahashi, J. Phys. Soc. Jpn. 52, 2210 (1983) 